\title{
RAS Mutational Status Detection in Tissue, Plasma, and Stool Samples for Colorectal Cancer
}

\author{
Liuying Zhu, ${ }^{1,2,3}$ Yuanhe Wang, ${ }^{1,2,3}$ Yang Zhou, ${ }^{1,2,3}$ Qian Dong $\mathbb{D}^{1,2,3}$ Yunpeng Liu $\mathbb{D}^{1,2,3}$ \\ and Jingdong Zhang $\mathbb{D}^{1,2,3}$ \\ ${ }^{1}$ Medical Oncology Department of Gastrointestinal Tumors, Liaoning Cancer Hospital \& Institute, Cancer Hospital of China \\ Medical University, No. 44, Xiaohe Road, Dadong District, Shenyang, 110042 Liaoning Province, China \\ ${ }^{2}$ Department of Medical Oncology, The First Hospital of China Medical University, Shenyang, China \\ ${ }^{3}$ Key Laboratory of Anticancer Drugs and Biotherapy of Liaoning Province, The First Hospital of China Medical University, \\ Shenyang, China
}

Correspondence should be addressed to Yunpeng Liu; ypliu@cmu.edu.cn and Jingdong Zhang; jdzhang@cancerhosp-ln-cmu.com Received 22 July 2019; Accepted 21 March 2020; Published 13 April 2020

Guest Editor: Qiang Tong

Copyright (C) 2020 Liuying Zhu et al. This is an open access article distributed under the Creative Commons Attribution License, which permits unrestricted use, distribution, and reproduction in any medium, provided the original work is properly cited.

Objective. RAS gene testing on tumor tissue biopsies is required for metastatic colorectal cancer (CRC) patients. However, it is infeasible for patients after curative surgery and repeated biopsy. This study is aimed at evaluating the consistency of $R A S$ genes in patient's plasma, stool, and tumor tissue samples, to explore whether plasma and stool samples can supplement or replace tumor tissue to assess baseline RAS gene status. Methods. Between June 2016 and October 2017, 53 patients with stage I-IV CRC from the Liaoning Cancer Hospital and the Department of Medical Oncology of the First Hospital of China Medical University were enrolled in the study. Patient tissues, peripheral blood, and stool samples were collected, and RAS gene tests were performed. Results. Analysis of the KRAS gene in tissue, plasma, and stool samples from $53 \mathrm{CRC}$ patients detected 25 cases (47\%) of KRAS gene mutations in the tissue samples, 20 cases (38\%) of KRAS gene mutations in plasma, and $18(34 \%)$ KRAS gene mutations in fecal samples. The overall consistency of KRAS gene status between tissue samples and plasma samples was $77.4 \%(p \leq 0.05)$ and between tissue samples and stool samples was $83 \%(p \leq 0.05)$. In stage IV cases, the agreement of KRAS gene status between tissue and plasma samples was 93.8\% $(p \leq 0.05)$ and $93.8 \%$ $(p \leq 0.05)$ between tissue and stool samples. Conclusion. There was a high overall consistency in KRAS mutational assessment between plasma, stool, and tissue samples. In stage IV patients, the consistency of KRAS gene detection between tissue and stools or plasma was higher.

\section{Introduction}

Nearly one million new cases of colorectal cancer (CRC) are diagnosed worldwide each year $[1,2]$, and it is one of the leading causes of cancer-related death [3-5]. However, in recent years, due to improvements in early detection and comprehensive treatment, the median overall survival (OS) has reached 30 months or longer [6-9].

Only CRC patients with wild-type RAS have benefited from anti-Epidermal Growth Factor Receptor (EGFR) treatment. As such, rapid and accurate detection of RAS gene mutations is vital for personalized CRC treatment. The use of tumor tissue to detect the RAS gene is the current gold standard in clinical practice and is also a frequently used detection indicator in clinical practice $[10,11]$.

The detection of circulating tumor DNA (ctDNA) is a minimally invasive detection method, which has obvious advantages over traditional tissue detection and is also more representative of tumor heterogeneity. ctDNA is derived from tumor cells, and the false positive detection for ctDNA is lower than that of protein biomarkers $[12,13]$. It has been reported that the average half-life of fetal DNA is 16.3 
minutes (with range 4-30 minutes) and that it is effectively eliminated within 2 hours [14]. Similarly, the half-life of ctDNA is also short $[15,16]$ and it allows us to monitor the dynamics of the tumor in units of hours, instead of weeks or even months, unlike tissue biopsies [17].

In recent years, noninvasive multitarget fecal DNA (mt-sDNA) detection technology has gradually matured. Tumor cells shed on the surface of precancerous lesions and are released into the feces. Through direct histological observation, it is found that shedding of tumor cells is a continuous process and is more frequent than that of ordinary epithelial cells. This may be due to excessive proliferation, which reduces cell-to-cell or cell-to-basement membrane adhesion [18]. This consistent tumor cell shedding allows us to monitor tumor-derived fecal DNA in a single stool sample. mt-sDNA testing can be used to detect 11 biomarkers (seven of which are DNA mutations in the $K R A S$ gene) in stool samples, and there is a high consistency in results between fecal testing and tissue testing [19-21]. This study is aimed at investigating the consistency of RAS gene detection in plasma, stool, and tumor tissue in patients with CRC.

\section{Material and Methods}

2.1. Study Design. In this study, we compared the consistency of RAS gene detection in plasma, stool, and tumor tissue in patients with CRC. All participants were enrolled between June 2016 and October 2017 in the Liaoning Cancer Hospital and the Department of Medical Oncology of the First Hospital of China Medical University. Informed written consent was obtained from all patients enrolled in this study. This study was approved by the Liaoning Cancer Hospital Institutional Review Board.

2.1.1. Diagnosis and Inclusion Criteria. Patients recruited met the following criteria: diagnosed with CRC by histopathology, aged between 18 and 80 years old, agreed to conduct histological $R A S$ gene testing, were willing to provide blood and stool samples in a tumor-bearing state, never received antiEGFR therapy, and signed informed consent.

2.2. Clinical Procedures. Patient peripheral blood and stool samples were collected from the remainder of routine admission examination samples. Peripheral blood samples of $5 \mathrm{ml}$ per patient were taken, placed in a blood collection tube supplemented with ethylenediaminetetraacetic acid, and sent to the laboratory within 4 hours. Here, samples were centrifuged at $3000 \mathrm{rpm}$ at $4^{\circ} \mathrm{C}$, and the plasma supernatant was collected and sealed in a low-adsorption EP tube for cryopreservation at $-80^{\circ} \mathrm{C}$. Stool samples (preferably dry) of $1 \mathrm{~g}$ were sealed in cryotubes and sent to the laboratory within 2 hours for cryopreservation at $-80^{\circ} \mathrm{C}$.

Amongst the 53 patients enrolled in this study, 49 patients were naive, while four had systemic treatment (2 with chemotherapy plus bevacizumab and 2 with chemotherapy alone).

2.3. Polymerase Chain Reaction (PCR). We used the QIAamp DNA Mini Kits (QIAGEN 51306) and the QIAamp DNA
TABLE 1

\begin{tabular}{lccc}
\hline & \multicolumn{3}{c}{ Tissue KRAS status } \\
\hline \multirow{3}{*}{ Plasma/stool KARS status } & Mutated & A & No mutated \\
& No mutated & C & D \\
\hline
\end{tabular}

Stool Mini Kit (QIAGEN 51504) to extract DNA from the peripheral blood and stool samples, respectively. The DNA quality was considered sufficient when sample DNA concentration $\geq 5 \mathrm{ng} / \mu \mathrm{l}$ and OD260/OD280 $=1.4-2.0$. The Typer software was used to interpret the molecular weight peaks detected by mass spectrometry, which were transformed to show the molecular weight peaks corresponding to single-nucleotide polymorphism sites.

Genetic testing of histology samples was performed using the standard procedures validated by each hospital, and the reported data were used for this investigation.

2.4. Statistical Analysis. Statistical analyses for paired sample detection were performed using SPSS software (SPSS version 25, SPSS Inc.).

Groups and formulae for analysis were specified as follows (Table 1): KRAS mutations in tissue and plasma or stool, A; KRAS mutations in plasma or stool but not tissue, $\mathrm{B}$; KRAS mutations in tissue but not in plasma or stool, C; and no KRAS mutations in tissue and plasma or stool, D. Positive percentage agreement $(\mathrm{PPA})=\mathrm{A} /(\mathrm{A}+\mathrm{C})$; negative percentage agreement $(\mathrm{NPA})=\mathrm{D} /(\mathrm{B}+\mathrm{D})$; overall agreement $=(\mathrm{A}+\mathrm{D}) /(\mathrm{A}+\mathrm{B}+\mathrm{C}+\mathrm{D})$.

\section{Results}

3.1. Patient Characteristics and Gene Test Results. Between June 2016 and October 2017, fifty-three CRC patients were enrolled, including 37 males (69.8\%) and 16 females (30.2\%). There were 46 patients $(86.8 \%)$ with the primary tumor located in the left side colorectum and seven patients (13.2\%) with the primary tumor in the right side colon. For the purpose of this study, the colon is divided into the left side colorectum and right side colon at the splenic flexure. Patient tumor stages were as follows: seven (13.2\%) stage I, $13(24.5 \%)$ stage II, 17 (32\%) stage III, and 16 (30.3\%) with stage IV. At the time of sample collection, no patients had received anti-EGFR-targeted therapy, 49 (92.4\%) patients had not received treatment, and four (7.6\%) patients with metastatic CRC had received therapy (two of these were chemotherapy plus bevacizumab and the others were chemotherapy alone). Patient parameters are summarized in Table 2.

The median concentration of plasma ctDNA was $8 \mathrm{ng} / \mu \mathrm{l}$. There was no significant correlation between plasma DNA concentration and the level of carcinoembryonic antigen $(r=0.2266)$. The median concentration of DNA extracted from feces was $62 \mathrm{ng} / \mu \mathrm{l}$. The stool DNA concentration was moderately correlated with the value of carcinoembryonic antigen $(r=0.6721)$. 
TABLE 2: Patient characteristics $(n=53)$.

\begin{tabular}{lcc}
\hline Characteristics & Cases $(n)$ & Percentage (\%) \\
\hline Gender & 37 & 69.8 \\
$\quad$ Male & 16 & 30.2 \\
Female & & \\
Tumor site & 46 & 86.8 \\
$\quad$ Left side colorectum & 7 & 13.2 \\
$\quad$ Right side colon & & \\
Clinical stage AJCC 8.0 & 7 & 13.2 \\
I & 13 & 24.5 \\
II & 17 & 32 \\
III & 16 & 30.3 \\
IV & & \\
Distant metastatic sites & 6 & 37.5 \\
1 & 5 & 31.2 \\
2 & 5 & 31.2 \\
$\quad \geq 3$ & & \\
Treatment before sampling & 49 & 7.6 \\
Untreated & 4 & \\
Treated & & \\
\hline
\end{tabular}

TABLE 3: Gene and mutation sites.

\begin{tabular}{|c|c|c|}
\hline Test gene & Codon & Mutation \\
\hline \multirow{5}{*}{ KRAS } & KRAS-Exon2 & G12S, G12D \\
\hline & KRAS-Exon 2 & $\begin{array}{l}\text { G12C, G12R, G12V, } \\
\text { G12A, G13C }\end{array}$ \\
\hline & KRAS-Exon2 & G13D \\
\hline & KRAS-Exon3 & Q61L, Q61R, Q61H \\
\hline & KRAS-Exon 4 & K117N, A146T, A146V, A146P \\
\hline \multirow{5}{*}{ NRAS } & NRAS-Exon 2 & G12D, G12S \\
\hline & NRAS-Exon 2 & G13D \\
\hline & NRAS-Exon 2 & G13R, G12C, G12V, G12A, G13V \\
\hline & NRAS-Exon3 & Q61R, Q61K, Q61L, Q61H \\
\hline & NRAS-Exon4 & A146T \\
\hline$B R A F$ & $B R A F$-Exon 15 & V600E \\
\hline
\end{tabular}

TABLE 4: Gene mutation rates.

\begin{tabular}{lccc}
\hline & Tissue (\%) & Plasma (\%) & Stool (\%) \\
\hline KRAS mutations & $25(47)$ & $19(35.8)$ & $19(35.8)$ \\
BRAF mutations & $6(11.3)$ & $6(11.3)$ & $3(5.7)$ \\
\hline
\end{tabular}

A total of 29 mutation sites were tested for and are shown in Table 3. Table 4 shows the total RAS mutations detected in patient samples. Of the 53 patients, 25 patients $(47 \%)$ had KRAS gene mutations detected in tumor tissue, 19 patients (35.8\%) from plasma, and 19 patients (35.8\%) from stool. No NRAS mutations were detected from tissue, plasma, and fecal samples. BRAF gene mutations were detected in tissue and plasma from six patients $(11.3 \%)$ and in stool from three patients $(5.7 \%)$.
TABLE 5: Agreement of KRAS status.

\begin{tabular}{lcccc}
\hline Concordance & PPA (\%) & NPA (\%) & $\begin{array}{c}\text { Overall } \\
\text { agreement } \\
(\%)\end{array}$ & $p$ \\
\hline $\begin{array}{l}\text { Plasma \& } \\
\text { tissue }\end{array}$ & $\begin{array}{l}64 \\
(16 / 25)\end{array}$ & $\begin{array}{c}89.3 \\
25 / 28)\end{array}$ & $77.4(41 / 53)$ & $p \leq 0.05$ \\
Stool \& tissue & 72 & 96.4 & $84.9(45 / 53)$ & $p \leq 0.05$ \\
\hline
\end{tabular}

TABLE 6: The agreement of KRAS gene status between plasma combined with stool and tissue samples. Data presented as patient numbers.

\begin{tabular}{lcccc}
\hline & & \multicolumn{2}{c}{ Tissue } & \\
& & Mutated & Unmutated & Total \\
\hline \multirow{3}{*}{ Plasma+stool } & Mutated & 24 & 4 & 28 \\
& Unmutated & 1 & 24 & 25 \\
& Total & 25 & 28 & 53 \\
\hline
\end{tabular}

\section{Patient Characteristics and the Agreement of KRAS Gene Mutation Detection in Plasma versus Tissue}

For the 25 patients in whose tissue sample KRAS mutation was detected, 16 also had a KRAS mutation detected in plasma (PPA of 64\%) and 18 in stool (PPA of 72\%). Amongst the 28 patients determined to be KRAS wild type (wt) from tissue analysis, 25 also had KRAS wt in plasma (NPA of 89.3\%) and 27 also had KRAS wt in stool samples (NPA of 96.4\%). The overall agreement between KRAS status between plasma and tissue samples was $77.4 \%$ (41/53 patients, $p \leq 0.05)$ and between stool and tissue samples was $84.9 \%$ (45 of 53 patients, $p \leq 0.05$ ). These results are shown in Table 5.

A combined blood and stool metric (plasma+stool), considered positive if a RAS mutation is detected in either, improves the PPA with tissue samples. Here, the PPA was $96 \%$ (24/25 patients), the NPA was $85.7 \%$ (24/28 patients), and the overall agreement rate was 48 in 53 patients $(90.6 \%, p \leq 0.05)$. These results are shown in Table 6 .

\section{KRAS Mutation Detection in Patients Separated by Tumor Stage}

We investigated the agreement between KRAS status of plasma and tissue samples, according to the clinical stage of the cancer. Stages I, II, III, and IV gave overall agreements of $28.6 \%(2 / 7, p>0.05), 69.2 \%(9 / 13, p>0.05), 88.2 \%$ $(15 / 17, p \leq 0.05)$, and $93.8 \%(15 / 16, p \leq 0.05)$. The same analysis for stool and tissue samples gave overall agreements of $57.1 \%(4 / 7, p>0.05), 84.6 \%(11 / 13, p>0.05), 88.2 \%$ $(15 / 17, p \leq 0.05)$, and $93.8 \%(15 / 16, p \leq 0.05)$ for stages I, II, III, and IV, respectively. These results are summarized in Table 7. 
TABLE 7: The agreement of mutant RAS detection from plasma, stool, and tissue samples in stage I to IV patients.

\begin{tabular}{lcccccc}
\hline \multirow{2}{*}{ Stage } & \multicolumn{2}{c}{ PPA } & \multicolumn{2}{c}{ NPA } & \multicolumn{2}{c}{ Overall agreement } \\
& Plasma \& tissue & Stool \& tissue & Plasma \& tissue & Stool \& tissue & Plasma \& tissue & Stool \& tissue \\
\hline I & $33.3 \%(2 / 6)$ & $50 \%(3 / 6)$ & $0 \%(0 / 1)$ & $100 \%(1 / 1)$ & $28.6 \%(2 / 7, p>0.05)$ & $57.1 \%(4 / 7, p>0.05)$ \\
II & $66.7 \%(4 / 6)$ & $85.7 \%(6 / 7)$ & $71.4 \%(5 / 7)$ & $85.7 \%(6 / 7)$ & $69.2 \%(9 / 13, p>0.05)$ & $84.6 \%(11 / 13, p>0.05)$ \\
III & $75 \%(6 / 8)$ & $75 \%(6 / 8)$ & $100 \%(9 / 9)$ & $100 \%(9 / 9)$ & $88.2 \%(15 / 17, p \leq 0.05)$ & $88.2 \%(15 / 17, p \leq 0.05)$ \\
IV & $80 \%(4 / 5)$ & $80 \%(4 / 5)$ & $100 \%(11 / 11)$ & $100 \%(11 / 11)$ & $93.8 \%(15 / 16, p \leq 0.05)$ & $93.8 \%(15 / 16, p \leq 0.05)$ \\
\hline
\end{tabular}

TABLE 8: The agreement between KRAS mutation status from plasma or stool and tissue samples, based on the sidedness of CRC.

\begin{tabular}{lcccccc}
\hline \multirow{2}{*}{ Site } & \multicolumn{2}{c}{ PPA } & \multicolumn{2}{c}{ NPA } & \multicolumn{2}{c}{ Overall agreement } \\
& Plasma \& tissue & Stool \& tissue & Plasma \& tissue & Stool \& tissue & Plasma \& tissue & Stool \& tissue \\
\hline Left side colorectum & $65 \%(13 / 20)$ & $85 \%(17 / 20)$ & $88.5 \%(23 / 26)$ & $96.2 \%(25 / 26)$ & $73.9 \%(36 / 46, p \leq 0.05)$ & $91.3 \%(42 / 46, p \leq 0.05)$ \\
Right side colon & $60 \%(3 / 5)$ & $20 \%(1 / 5)$ & $100 \%(2 / 2)$ & $100 \%(2 / 2)$ & $71.4 \%(5 / 7, p>0.05)$ & $42.9 \%(3 / 7, p>0.05)$ \\
\hline
\end{tabular}

\section{Comparison of KRAS Mutation Status in Blood, Stool, and Tissue Samples from Primary Tumors of the Left Side Colorectum and Right Side Colon}

In the study population, there were 46 patients with left-sided CRC and seven patients with right-sided CRC. The overall agreement between plasma and tissue samples was similar in left- and right-sided CRCs $(73.9 \%$ and $71.4 \%$, respectively). However, while the overall agreement between fecal and tissue sample KRAS detection was $91.3 \%(42 / 46)$ in left-sided CRC, it was just $42.9 \%$ (3/7) in right-sided CRC. These results are shown in Table 8.

\section{Consistency of BRAF Gene Detection in Patient Samples}

In six patients, BRAF mutation was detected in tissue samples. In four of these patients, BRAF mutation was also detectable in plasma (PPA of 66.7\%) and four had a BRAF mutation in stool (PPA of 66.7\%). Of the 47 patients with wild-type BRAF detected in tissue samples, 46 also had wild-type BRAF detected in ctDNA (NPA of 97.5\%) and 47 also had a BRAF wt (NPA of 100\%). The overall agreement of $B R A F$ mutational status between plasma and tissue samples was $94.3 \%$ (50/53 patients, $p \leq 0.05)$ and between stool and tissue samples was $96.2 \%$ (51/53 patients, $p \leq 0.05)$. These findings are shown in Tables 9 and 10 for plasma and stool comparisons, respectively.

\section{Discussion}

As only CRC patients with wild-type RAS genes benefit from anti-EGFR treatment, it is important to detect the RAS gene accurately and conveniently. Gene detection from tumor biopsy tissue is the gold standard, but repeating tissue biopsy by enteroscopy may increase related complications $[22,23]$ and only reflect the state of the genome at a certain site and time. Many patients have distant metastases at the time of diagnosis, and tissue biopsy specimens cannot be obtained
TABLE 9: Agreement in detection rates between plasma and tissue samples for $B R A F$.

\begin{tabular}{lcccc}
\hline & \multicolumn{4}{c}{ Tissue BRAF status } \\
\hline \multirow{3}{*}{ Plasma BRAF } & Mutated & Unmutated & Total \\
status & Mutated & 4 & 1 & 5 \\
& Unmutated & 2 & 46 & 48 \\
& Total & 6 & 47 & 53 \\
\hline
\end{tabular}

TABLE 10: Agreement in detection rates between plasma and stool samples for BRAF.

\begin{tabular}{lcccc}
\hline & \multicolumn{4}{c}{ Tissue BRAF status } \\
\hline \multirow{4}{*}{ Stool BRAF status } & Mutated & Unmutated & Total \\
& Mutated & 4 & 0 & 4 \\
& Unmutated & 2 & 47 & 49 \\
& Total & 6 & 47 & 53 \\
\hline
\end{tabular}

by surgery in these cases [24,25]. Here, we investigate whether plasma and stool samples can be used as a supplement or alternative to tumor tissue for RAS gene status testing.

As early as 1992, Sidransky et al. published an article in Science, which reported that the agreement rate of KRAS mutation in feces and tumor tissues was 89\% (8/9) [26]. Similarly, a study by Vidal et al. showed that the overall agreement of RAS mutation status between ctDNA and tissue samples was $93 \%(107 / 115)$, with a positive agreement rate of $96.4 \%$ and a negative agreement rate of $90 \%$ [27]. In this study, plasma, fecal, and tissue samples were simultaneously included in comparative analysis. The high agreement between tissue and fecal or plasma KRAS gene assays supports the use of peripheral blood or feces as a viable complement or alternative to tissue DNA testing. The results also showed that combining KRAS gene detection results from plasma and stool samples increased the positive rate of detection $(96 \%, p \leq 0.05)$, indicating that these two metrics can complement each other. 
When tumor cells are shed, or once cells become apoptotic, small fragments of DNA are released into the circulatory system. Mutations in this ctDNA have been detected in almost all types of cancer, and the later the tumor stage or the higher the malignancy of the tumor, the higher the frequency of mutations detected by ctDNA. The same is true for DNA testing in stool samples. Anti-EGFR treatment may have some impact on the status of the RAS gene; at the time of sample collection, all patients had not received antiEGFR-targeted therapy. 49 (92.4\%) patients had not received antineoplaston, $4(7.6 \%)$ patients with $\mathrm{mCRC}$ had received therapy ( 2 of them were chemotherapy+bevacizumab and the others were chemotherapy alone). With tumor remission after treatment, gene abundance in plasma DNA is affected. The gene abundance of RAS wild type is relatively sensitive; it may decrease below the detection line after chemotherapy, making it undetectable; and the RAS wild type may also change to RAS mutant type after treatment. However, in this study, the 4 treated patients had the same RAS gene status. In this study, patients were grouped according to AJCC $8.0 \mathrm{can}$ cer stages and analyzed separately. The agreement between sample mutation status readouts was higher in patients with stages IV and III than in patients with stages I and II. However, the agreement between feces and tissue in stages I and II was significantly higher than that in plasma and tissue ( $57.1 \%$ to $28.6 \%$ in stage I and $84.6 \%$ to $69.2 \%$ in stage II, for feces and plasma, respectively). In colonic tumors, whether it is a malignant tumor or an advanced adenoma, exfoliated cells detached from their surface can be released into the feces directly. Therefore, in early cancer stages, tumor DNA in stool samples may be easier to detect than ctDNA and may be a better choice than blood sample analysis for early screening.

It has been reported that mt-sDNA testing has a similar consistency rate for left- and right-sided CRCs [28]. The right side colon has a large intestinal lumen and a thin intestinal wall and expands easily. Its physiological function is to absorb water, electrolytes, and some glucose. As such, the contents of the right-sided colon are mostly liquid or semiliquid, which affects the purity of tumor DNA extraction in the feces. In contrast, the left-sided colorectum has a narrow intestinal lumen and its main physiological function is to absorb water and store stools. Therefore, the contents of the left-sided colorectum are relatively dry, which are conducive to tumor DNA extraction and gene detection. The data here show that blood test results are not affected by the location of the primary tumor site (with left side colorectum agreement with tissue status at $73.9 \%$ and right side colon at $71.4 \%$ ) but that fecal testing performs better in the left side colorectum $(91.3 \%$ agreement compared with $42.9 \%$ in the right side colon).

The sample size in this study is relatively small; however, larger studies are required to validate these results.

\section{Conclusion}

The high agreement between plasma-, fecal-, and tissuebased detection of RAS mutational status supports the use of plasma and fecal RAS gene detection as a viable alternative to tissue detection in CRC patients. The detection accuracy from plasma and fecal samples is higher for later stage CRCs, while for early stage screening, analysis of fecal samples may be a better choice.

\section{Data Availability}

The data used to support the findings of this study are included within the article.

\section{Conflicts of Interest}

I declare that none of the authors have a conflict of interest.

\section{Acknowledgments}

We thank Edanz Group (https://en-author-services .edanzgroup.com/) for editing a draft of this manuscript. This research was funded by the Science and Technology Planning Project of Shenyang (Nos. 191124088 and F15139-9-27), the Science and Technology Planning Project of Liaoning Province of China (No. 201800449), the National Key R\&D Program of China (Grant \#2018YFC1311600), and the scientific research foundation for the introduction of talents, Liaoning Cancer Hospital \& Institute (No. Z1702).

\section{References}

[1] Global Burden of Disease Cancer Collaboration, "The global burden of cancer 2013," JAMA Oncology, vol. 1, no. 4, pp. 505-527, 2015.

[2] A. B. Benson 3rd., "Epidemiology, disease progression, and economic burden of colorectal cancer," Journal of Managed Care Pharmacy, vol. 13, no. 6, Supplement C, pp. S5-S18, 2007.

[3] A. Jemal, F. Bray, M. M. Center, J. Ferlay, E. Ward, and D. Forman, "Global cancer statistics," CA: a Cancer Journal for Clinicians, vol. 61, no. 2, pp. 69-90, 2011.

[4] J. Ferlay, E. Steliarova-Foucher, J. Lortet-Tieulent et al., "Cancer incidence and mortality patterns in Europe: estimates for 40 countries in 2012," European Journal of Cancer, vol. 49, no. 6, pp. 1374-1403, 2013.

[5] J. Ferlay, I. Soerjomataram, R. Dikshit et al., "Cancer incidence and mortality worldwide: sources, methods and major patterns in GLOBOCAN 2012," International Journal of Cancer, vol. 136, no. 5, pp. E359-E386, 2015.

[6] H. J. Schmoll, E. van Cutsem, A. Stein et al., "ESMO consensus guidelines for management of patients with colon and rectal cancer. A personalized approach to clinical decision making," Annals of oncology, vol. 23, no. 10, pp. 2479-2516, 2012.

[7] T. E. Botrel, L. G. Clark, L. Paladini, and O. A. Clark, "Efficacy and safety of bevacizumab plus chemotherapy compared to chemotherapy alone in previously untreated advanced or metastatic colorectal cancer: a systematic review and meta-analysis," BMC Cancer, vol. 16, no. 1, p. 677, 2016.

[8] R. F. Liang and L. L. Zheng, "The efficacy and safety of panitumumab in the treatment of patients with metastatic colorectal cancer: a meta-analysis from five randomized controlled trials," Drug Design, Development and Therapy, vol. 9, pp. 4471-4478, 2015. 
[9] R. Labianca, B. Nordlinger, G. D. Beretta et al., "Early colon cancer: ESMO Clinical Practice Guidelines for diagnosis, treatment and follow-up," Annals of Oncology, vol. 24, Supplement 6, pp. vi64-vi72, 2013.

[10] C. Montagut, A. Dalmases, and B. Bellosillo, "Identification of a mutation in the extracellular domain of the epidermal growth factor receptor conferring cetuximab resistance in colorectal cancer," Nature Medicine, vol. 18, no. 2, pp. 221223, 2012.

[11] S. Misale, R. Yaeger, S. Hobor et al., "Emergence of KRAS mutations and acquired resistance to anti-EGFR therapy in colorectal cancer," Nature, vol. 486, no. 7404, pp. 532-536, 2012.

[12] V. Vasioukhin, P. Anker, P. Maurice, J. Lyautey, C. Lederrey, and M. Stroun, "Point mutations of the N-ras gene in the blood plasma DNA of patients with myelodysplastic syndrome or acute myelogenous leukaemia," British Journal of Haematology, vol. 86, no. 4, pp. 774-779, 1994.

[13] G. D. Sorenson, D. M. Pribish, F. H. Valone, V. A. Memoli, D. J. Bzik, and S. L. Yao, "Soluble normal and mutated DNA sequences from single-copy genes in human blood," Cancer Epidemiology Biomarkers \& Prevention, vol. 3, pp. 67-71, 1994.

[14] N. Beije, J. C. Helmijr, M. J. Weerts et al., "Somatic mutation detection using various targeted detection assays in paired samples of circulating tumor DNA, primary tumor and metastases from patients undergoing resection of colorectal liver metastases," Molecular Oncology, vol. 10, no. 10, pp. 15751584, 2016.

[15] F. Diehl, M. Li, D. Dressman et al., "Detection and quantification of mutations in the plasma of patients with colorectal tumors," Proceedings of the National Academy of Sciences of the United States of America, vol. 102, no. 45, pp. 1636816373, 2005.

[16] G. Siravegna, B. Mussolin, M. Buscarino et al., "Clonal evolution and resistance to EGFR blockade in the blood of colorectal cancer patients," Nature Medicine, vol. 21, no. 7, pp. 795-801, 2015.

[17] M. Gerlinger, A. J. Rowan, S. Horswell et al., "Intratumor heterogeneity and branched evolution revealed by multiregion sequencing," The New England Journal of Medicine, vol. 366, no. 10, pp. 883-892, 2012.

[18] D. Ahlquist, J. J. Harrington, L. J. Burgart, and P. C. Roche, "Morphometric analysis of the "mucocellular layer" overlying colorectal cancer and normal mucosa: relevance to exfoliation and stool screening," Human Pathology, vol. 31, no. 1, pp. 51$57,2000$.

[19] D. G. Redwood, E. D. Asay, I. D. Blake et al., "Stool DNA testing for screening detection of colorectal neoplasia in Alaska native people," Mayo Clinic Proceedings, vol. 91, no. 1, pp. 61-70, 2016.

[20] "Multitarget stool DNA testing for colorectal-cancer screening," The New England Journal of Medicine, vol. 370, no. 14, pp. 1287-1297, 2014.

[21] S. M. A. Kormi, S. Ardehkhani, and M. A. Kerachian, "New insights into colorectal cancer screening and early detection tests," Colorectal Cancer, vol. 6, no. 2, pp. 63-68, 2017.

[22] W. S. Atkin, R. Edwards, I. Kralj-Hans et al., "Once-only flexible sigmoidoscopy screening in prevention of colorectal cancer: a multicentre randomised controlled trial," The Lancet, vol. 375, no. 9726, pp. 1624-1633, 2010.
[23] D. A. Lieberman, D. Ransohoff, S. J. Winawer, F. M. Giardello, D. A. Johnson, and T. R. Levin, "Guidelines for colonoscopy surveillance after screening and polypectomy: a consensus update by the US multi-society task force on colorectal cancer," Gastroenterology, vol. 143, no. 3, pp. 844-857, 2012.

[24] R. Nishihara, K. Wu, M. B. Lochhead et al., "Long-term colorectal cancer incidence and mortality after lower endoscopy," The New England Journal of Medicine, vol. 369, no. 12, pp. 1095-1105, 2013.

[25] L. Schlessinger and D. M. Eddy, “Archimedes: a new model for simulating health care systems-the mathematical formulation," Journal of Biomedical Informatics, vol. 35, no. 1, pp. 37-50, 2002.

[26] D. Sidransky, T. Tokino, and S. R. Hamilton, "Identification of ras oncogene mutations in the stool of patients with curable colorectal tumors," Science, vol. 256, no. 5053, pp. 102-105, 1992.

[27] J. Vidal, L. Muinelo, A. Dalmases et al., "Plasma ctDNA RAS mutation analysis for the diagnosis and treatment monitoring of metastatic colorectal cancer patients," Annals of Oncology, vol. 28, no. 6, pp. 1325-1332, 2017.

[28] H. Brenner, M. Hoffmeister, V. Arndt, C. Stegmaier, L. Altenhofen, and U. Haug, "Soluble normal and mutated DNA sequences from single-copy genes in human blood," Journal of the National Cancer Institute, vol. 102, no. 2, pp. 89-95, 2010. 